\title{
The Predictive Value of Tissue Doppler Indices for Early Recurrence of Atrial Fibrillation After Electrical Cardioversion
}

This article was published in the following Dove Press journal: Clinical Interventions in Aging

\author{
Rasa Karaliute ${ }^{1,2}$ \\ Justina Jureviciute ${ }^{2}$ \\ Julija Jurgaityte ${ }^{2}$ \\ Agne Rimkute ${ }^{2}$ \\ Vaida Mizariene ${ }^{2}$ \\ Giedre Baksyte ${ }^{2}$ \\ Tomas Kazakevicius ${ }^{2}$ \\ Daiva Urboniene ${ }^{3}$ \\ Ausra Kavoliuniene ${ }^{2}$ \\ 'Laboratory of Behavioral Medicine, \\ Neuroscience Institute, Lithuanian \\ University of Health Sciences, Kaunas, \\ Lithuania; ${ }^{2}$ Department of Cardiology, \\ Lithuanian University of Health Sciences, \\ Kaunas, Lithuania; ${ }^{3}$ Department of \\ Laboratory Medicine, Lithuanian \\ University of Health Sciences, Kaunas, \\ Lithuania
}

Correspondence: Rasa Karaliute Laboratory of Behavioral Medicine, Neuroscience Institute, Lithuanian University of Health Sciences, Eiveniu St.

4, Kaunas LT-5016I, Lithuania

$\mathrm{Tel}+37067766157$

Email rasa.karaliute@Ismuni.It
Purpose: Spectral tissue Doppler-derived E/e' ratio has been proposed as the best parameter for prediction of atrial fibrillation (AF). Relaxation and contraction are equivalent parts of a continuous cardiac cycle, where systolic and diastolic abnormalities have a variable contribution to the left ventricle (LV) failure. The aim of this study was to investigate whether the $\mathrm{E} /\left(\mathrm{e}^{\prime} \mathrm{xs}\right.$ ') ratio is a better index than $\mathrm{E} / \mathrm{e}^{6}$ to predict $\mathrm{AF}$ recurrence and to determine the changes of spectral tissue Doppler indices 1 month after the electrical cardioversion (ECV).

Patients and Methods: The study included 77 persistent AF patients with restored sinus rhythm (SR) after ECV. Only patients with normal LV ejection fraction (EF) were included. Echocardiography and NT-proBNP laboratory findings were performed. A primary outcome was the early (within 1 month) recurrence of AF.

Results: After a 1 month follow-up period, 39 patients $(50.6 \%)$ were in SR. E/e' $(\mathrm{HR}=1.74$, $P=0.001)$ and $\mathrm{E} /\left(\mathrm{e}^{\prime} \times \mathrm{s}^{\prime}\right)$ ratios $(\mathrm{HR}=8.17, P=0.01)$ were significant predictors of $\mathrm{AF}$ recurrence. $\mathrm{E} /\left(\mathrm{e}^{\prime} \times \mathrm{s}\right.$ ') in combination with $\mathrm{LV}$ end-diastolic diameter $>49.3 \mathrm{~mm}$ and NT-proBNP $>2000 \mathrm{ng} / \mathrm{L}$ demonstrated a higher contribution in the model to predict $\mathrm{AF}$ recurrence compared to the $\mathrm{E} / \mathrm{e}$ ' ratio (18.94, $P=0.005$ vs $1.95, P=0.001)$. On ROC analysis, $\mathrm{E} /\left(\mathrm{e}^{\prime} \times \mathrm{s}\right.$ ') and $\mathrm{E} / \mathrm{e}^{\prime}$ showed similar diagnostic accuracy $\left(\mathrm{E} /\left(\mathrm{e}^{\prime} \times \mathrm{s}^{\prime}\right), \mathrm{AUC}=0.71, P=0.002\right.$ and $\mathrm{E} / \mathrm{e}^{\prime}$, $\mathrm{AUC}=0.75, P<0.0001)$. Average $\mathrm{e}^{6}$ value significantly decreased after 1 month in SR (from $10.76 \pm 1.24$ to $8.96 \pm 1.47 \mathrm{~cm} / \mathrm{s}, P=0.01$ ), E wave did not change significantly and $\mathrm{E} / \mathrm{e}$ ' ratio tended to improve. A decrease of average $\mathrm{e}^{\text {' }}$ and an increase of average s' values led to significant improvement of E/(e'xs') ratio.

Conclusion: E/(e'xs') and E/e' ratios are comparable to predict early AF recurrence after ECV in patients with persistent AF. The e' value decreased significantly after 1 month follow-up period after ECV for persistent AF patients.

Keywords: diastolic function, E/( $\left.\mathrm{e}^{6} \mathrm{xs}^{\mathrm{c}}\right), \mathrm{E} / \mathrm{e}^{\mathrm{s}}$, NT-proBNP, left ventricular end-diastolic diameter

\section{Introduction}

Atrial fibrillation (AF) and heart failure with preserved ejection fraction (EF) often coincide and are pathogenetically associated with each other, although the cause-effect relationship has not been fully determined. ${ }^{1}$ The ratio of mitral peak early diastolic filling velocity (E) to peak early diastolic mitral annular velocity (e') estimated by Doppler tissue imaging $\left(E / e^{\triangleleft}\right)$ is one of the four main echocardiographic variables for diagnosing a diastolic dysfunction. ${ }^{2,3}$ In recent studies, a new Doppler index - the ratio of $\mathrm{E}$ to $\mathrm{e}^{\text {' }}$ multiplied by peak systolic mitral annular velocity (s') derived by Doppler 
tissue imaging $\left(E /\left(e^{\prime} x s^{\prime}\right)\right.$, is demonstrated to be superior to $E /$ e', e', s', or E in sinus rhythm (SR) patients and has high diagnostic accuracy, particularly in patients with intermediate values for E/e' ratio $(8-15) .{ }^{4}$ Elevated E/e' ratio and to a lesser extent E/(e'xs') have been confirmed as beneficial predictors of new-onset AF, AF recurrence after electrical cardioversion (ECV), and worse outcomes in patients with various heart diseases. $^{5-10}$ A comprehensive systematic review by $\mathrm{D}$. Kotecha reported that diastolic function indices, particularly E/e', are valid and reproducible in patients with AF when the appropriate cardiac cycles are carefully selected. ${ }^{11}$ Nevertheless, Park et al ${ }^{12}$ revealed in their small study that E/e' was significantly lower in AF compared with SR during 1 month after ECV in the same patients. There is limited data demonstrating how e" changes after SR restoration in AF patients and this information is useful to confirm

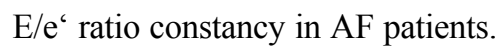

The aim of this study was to investigate whether the E/ (e'xs') ratio is a better index than $E / e^{\prime}$ to predict $A F$ recurrence and to determine the changes of spectral tissue Doppler indices 1 month after the ECV for persistent AF patients with normal left ventricular (LV) EF.

\section{Patients and Methods}

\section{Study Population and Data Collection}

This prospective study included 77 patients with persistent AF scheduled for ECV in the Cardiology Department at the Hospital of Lithuanian University of Health Sciences Kaunas Clinics during the 24 months screening period.

Inclusion criteria were as follows: age $>18$ years, $\mathrm{AF}>48$ hours, proven indication to restore SR by ECV, and obtained written informed consent. The exclusion criteria were: refusal to participate in the study, relevant medical history of present heart failure with preserved or reduced $\mathrm{EF}$, newly diagnosed significant $\mathrm{LV}$ dysfunction ( $\mathrm{LV} \mathrm{EF<35 \% ),} \mathrm{confirmed} \mathrm{ischemic} \mathrm{heart} \mathrm{disease,} \mathrm{severe}$ valvular heart disease, permanent pacing, and known other diseases which could affect results of laboratory findings (severe renal failure and liver disease, use of glucocorticosteroids, any type of cancer, thyroid disorders). A flowchart of patient selection is shown in Figure 1.

Primary outcome was the early (within 1 month) recurrence of AF. According to that, patients were assigned into two groups: those who maintained SR and those with relapse of AF diagnosed by clinical manifestations and ECG.
This study was conducted in accordance with the Declaration of Helsinki. The study protocol and a written informed consent form were reviewed and approved by the Kaunas Regional Biomedical Research Ethics Committee (ref. number BE-10-4). Every patient signed an informed consent form, which was provided to the patient with sufficient time to read and ask questions prior to signing.

\section{Biochemical Analyses}

Blood samples were collected immediately before ECV for all patients and repeated after 1 month in the case of SR maintenance, centrifuged, and stored at $-80^{\circ} \mathrm{C}$ until analysis. The plasma N-terminal proatrial natriuretic peptide (NTproANP) concentrations were estimated using Human NTproANP ELISA Kit (Biomedica Immunoassay, Biomedica Medizinprodukte GmbH \& Co KG, Austria). N-terminal-pro B-type natriuretic peptide (NT-ProBNP) concentrations were estimated using NT-ProBNP ELISA Kit (PathFast, LSI Medience Corporation, Japan). Following the Quality Specifications for B-Type Natriuretic Peptide Assays recommendations provided by the Committee on Standardization of Markers of Cardiac Damage of the IFCC the manufacturer's assay characteristics and results were recalculated from original units to $\mathrm{ng} / \mathrm{L}(1 \mathrm{nmol} / \mathrm{L}=12,700 \mathrm{ng} / \mathrm{L}$ for NTproANP and $1 \mathrm{pg} / \mathrm{mL}=1 \mathrm{ng} / \mathrm{L}$ for NT-proBNP). ${ }^{13}$ For the evaluation of renal function, serum creatinine levels were determined before ECV, and glomerular filtration rate was evaluated according to the CKD-EPI equation. ${ }^{14}$

\section{Electrical Cardioversion Procedure}

ECVs were performed using a biphasic defibrillator with energy selected on $200 \mathrm{~J}$ under short-term general sedation with propofol after obtaining patients informed consent. If SR was not restored, the ECV was repeated with increasing energy (by $100 \mathrm{~J}$ ) up to three repetitions. The decision of medical therapy was taken by a physician according to the current European Society of Cardiology (ESC) guidelines for the management of $\mathrm{AF}^{15}$

\section{Transthoracic Echocardiography}

Two-dimensional echocardiography was performed within 24 hours after ECV for all patients and repeated 1 month later in the case of successful SR maintenance using the diagnostic ultrasound system (EPIQ 7, Phillips Ultrasound, Inc. Washington, USA) by one investigator. All measurements were averaged over five cardiac cycles with simultaneously obtained ECG. Two-dimensional measurements 


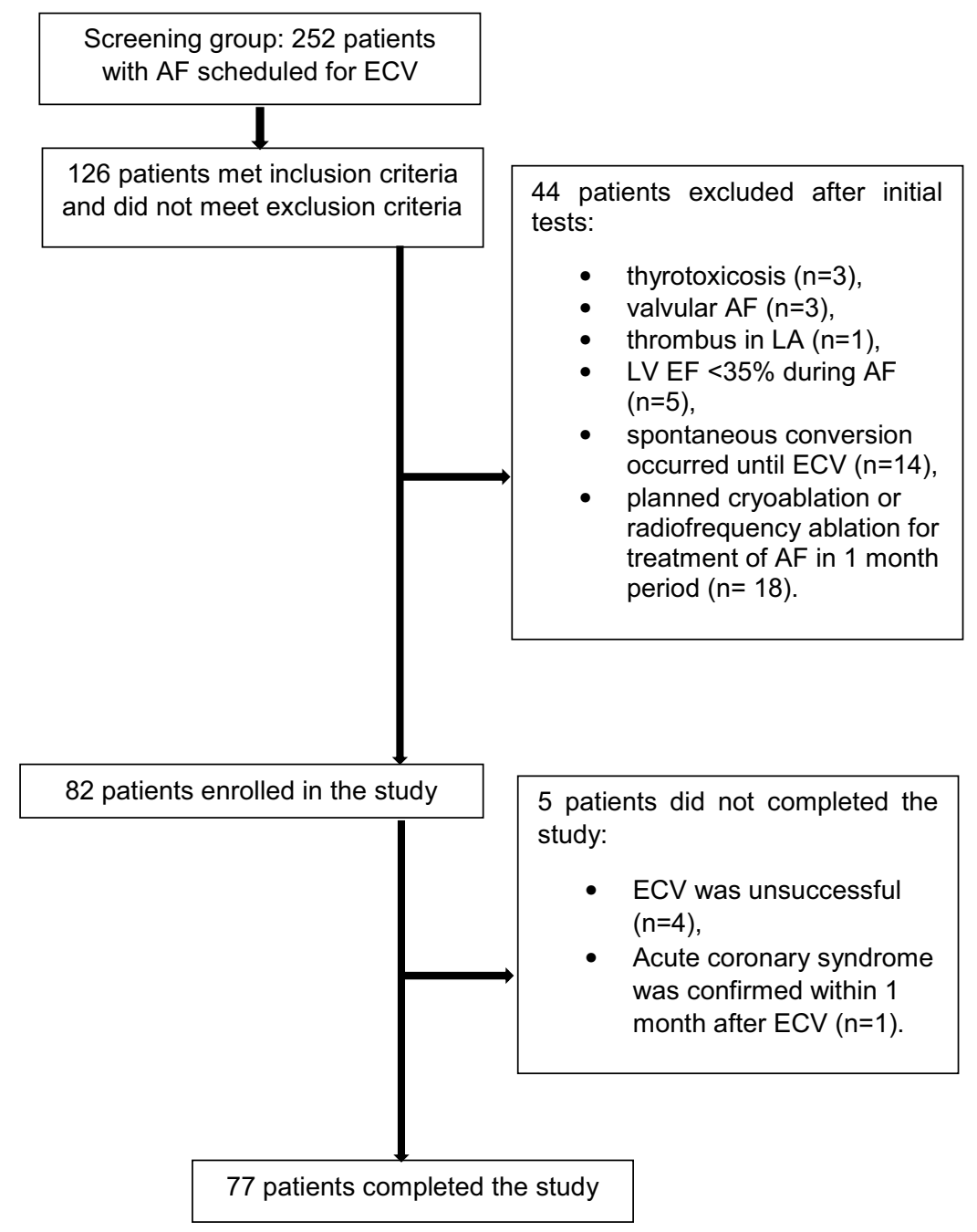

Figure I A flowchart of patient selection.

Abbreviations: AF, atrial fibrillation; $E C V$, electrical cardioversion; $L A$, left atrium; $L V E F$, left ventricular ejection fraction.

were performed according to the recommendations of the American Society of Echocardiography. ${ }^{16}$

Left atrium (LA) volumes were measured by the modified Simpson's disc summation method using apical fourand two-chamber views. LA maximum volume $\left(\mathrm{V}_{\max }\right)$ was measured at the end-systolic frame, just before the opening of the mitral valve. LA minimum volume $\left(\mathrm{V}_{\text {min }}\right)$ was measured at the end-diastolic frame, at the mitral valve closure. $\mathrm{V}_{\max }$ was indexed to the body surface area to derive the LA volume index (LAVI). Left ventricular EF was assessed by the modified Simpson's method. LA emptying fraction (LAEF) was calculated using the following equation $\left(\mathrm{V}_{\max }-\mathrm{V}_{\min }\right) / \mathrm{V}_{\max } \times 100 .{ }^{17}$

Mitral peak early (E) diastolic filling velocity was recorded from the apical four-chamber view with 3-mm PW Doppler sample volume placed between mitral leaflet tips. Tissue Doppler imaging was performed in the apical four-chamber view with a 5-mm PW Doppler sample volume at the lateral and septal basal regions. The peak septal and lateral early diastolic ( $\mathrm{e}^{\prime}$ wave) and the peak systolic (s' wave) mitral annular velocities were collected. The following ratios were calculated: E/e' and E/ $\left(e^{\prime} x s^{\prime}\right) .^{3}$

\section{Statistical Analysis}

Statistical analysis was performed by IBM $^{\circledR}$ SPSS $^{\circledR}$ Statistics 20 (Armonk, NY: IBM Corp., USA). All continuous variables were assessed by the KolmogorovSmirnov test and were normally distributed. They are expressed as mean \pm standard deviation. The categorical variables are expressed as absolute numbers (percentages). Normally distributed continuous variables were compared using independent-samples and paired-samples T-tests. A Mann-Whitney rank sum test was used if data was not 
normally distributed. Categorical variables were compared using the Chi-square test. Univariate binary logistic regression was carried out to identify the risk factors associated with AF recurrence and results are shown as a hazard ratio (HR) with 95\% confidence intervals (CI). Then we developed two multivariate models using the potential confounding factors. To minimize the problem of collinearity, E wave and average $\mathrm{e}^{\prime}$ values were not included, being already represented within the derived ratios, and these derived ratios $\left(E / e^{6}\right.$ and $\left.E /\left(e^{6} x^{6}\right)\right)$ were included in two separate models. Receiver-operating characteristic (ROC) analysis was used to determine optimal cutoff values with corresponding sensitivity and specificity of continuous variables for the prediction of AF recurrence. A $P$-value $<0.05$ was considered significant.

\section{Results}

\section{Clinical and Echo-Doppler Characteristics}

At 1-month follow-up period, 39 patients (50.6\%) were in SR. Patients with AF recurrence and maintained SR were similar in relation to age, sex, used medications, concentrations of natriuretic peptides, and cardiovascular risk factors; however, more patients from the SR group had diabetes mellitus (respectively, $9(24.3 \%)$ vs $2(5.3 \%)$, $P=0.02)$. More patients from the AF group had AF of longer duration (respectively 5 (2-24) vs 13 (4-47) months, $P=0.04$ ), however AF and SR groups did not differ significantly regarding the duration of the current AF episode. Baseline characteristics for the entire patients' population are reported in Table 1.

Patients enrolled in the study had a moderate-to-severe LA enlargement (maximum LAVI $52.86 \pm 10.47 \mathrm{~mL} / \mathrm{m}^{2}$, antero-posterior LA diameter $49.03 \pm 3.86 \mathrm{~mm}$ ). No significant difference in LAVI, cardiac dimensions, and LAEF was detected between the SR and AF groups. Patients from the AF group showed higher $\mathrm{E}$ wave (respectively, E wave $103.03 \pm 15.93$ vs $89.41 \pm 14.84 \mathrm{~cm} / \mathrm{s}, P=<0.0001)$, lower septal, lateral, and average $\mathrm{e}^{\prime}$ velocities (respectively, average $\mathrm{e}^{\prime}$ velocity $9.93 \pm 1.19$ vs $10.76 \pm 1.24 \mathrm{~cm} / \mathrm{s}$, $P=0.004$ ), and in turn higher septal $\mathrm{E} / \mathrm{e}^{\prime}$ (respectively, $10.47 \pm 1.73$ vs $8.49 \pm 2.03, P<0.0001)$ and $E /\left(e^{\prime} x s^{\prime}\right)$ ratios (respectively, $1.53 \pm 0.29$ vs $1.29 \pm 0.37, P=0.003$ ) when compared with patients who remained in SR. Baseline Echo-Doppler parameters of the study group are reported in Table 2.
Table I Baseline Characteristics of the Study Groups

\begin{tabular}{|c|c|c|c|c|}
\hline & $\begin{array}{l}\text { Total Cohort } \\
(n=77)\end{array}$ & $\begin{array}{l}\text { SR Group } \\
(n=39)\end{array}$ & $\begin{array}{l}\text { AF Group } \\
(n=38)\end{array}$ & $\mathbf{P}$ \\
\hline \multicolumn{5}{|c|}{ Clinical characteristics } \\
\hline Age, years & $63 \pm 10$ & $62 \pm 9$ & $65 \pm 10$ & 0.15 \\
\hline Males, n (\%) & $48(62.3)$ & $26(66.7)$ & $22(57.9)$ & 0.43 \\
\hline BMI, $\mathrm{kg} / \mathrm{m}^{2}$ & $29.52 \pm 4.09$ & $29.33 \pm 4.35$ & $29.72 \pm 3.85$ & 0.68 \\
\hline $\begin{array}{l}\text { eGFR, } \mathrm{mL} / \mathrm{min} / \\
1.73 \mathrm{~m}^{2}\end{array}$ & $69.45 \pm|5.7|$ & $65.50 \pm 14.96$ & $72.84 \pm 15.74$ & 0.06 \\
\hline $\mathrm{AH}, \mathrm{n}(\%)$ & $64(85.3)$ & $32(86.5)$ & $32(84.2)$ & 0.78 \\
\hline $\begin{array}{l}\text { Diabetes } \\
\text { mellitus, n (\%) }\end{array}$ & II (I4.7) & $9(24.3)$ & $2(5.3)$ & 0.02 \\
\hline Smokers, n (\%) & $13(18.3)$ & $7(20)$ & $6(16.7)$ & 0.72 \\
\hline $\begin{array}{l}\text { AF history, } \\
\text { months }\end{array}$ & $8(2-37)$ & $5(2-24)$ & $13(4-47)$ & 0.04 \\
\hline \multicolumn{5}{|c|}{ AF duration before ECV, $n(\%)$} \\
\hline$<3$ months & $37(48.1)$ & $22(56.4)$ & $15(39.5)$ & 0.32 \\
\hline $3-6$ months & $21(27.3)$ & $9(23.1)$ & $12(31.6)$ & \\
\hline $6-12$ months & $19(24.7)$ & $8(20.5)$ & II (28.9) & \\
\hline $\begin{array}{l}\text { First AF } \\
\text { episode, } n(\%)\end{array}$ & $35(45.5)$ & $20(51.3)$ & $15(39.5)$ & 0.29 \\
\hline \multicolumn{5}{|c|}{ Medical therapy, n (\%) } \\
\hline Beta-blockers & $70(94.6)$ & $33(47.1)$ & $37(52.9)$ & 0.28 \\
\hline ACEI or ARB & $56(75.7)$ & $28(77.8)$ & $28(73.7)$ & 0.68 \\
\hline AAD & $45(60.8)$ & $25(55.6)$ & $20(44.4)$ & 0.14 \\
\hline \multicolumn{5}{|l|}{ Biomarkers, ng/L } \\
\hline NT-proBNP & $\begin{array}{l}2304.48 \\
\pm 1570.65\end{array}$ & $\begin{array}{l}2334.25 \\
\pm 1570.39\end{array}$ & $\begin{array}{l}2274.71 \\
\pm 1592.59\end{array}$ & 0.87 \\
\hline NT-proANP & $\begin{array}{l}59,324.88 \pm \\
31,155.56\end{array}$ & $\begin{array}{l}66,140.54 \pm \\
36,999.74\end{array}$ & $\begin{array}{l}53,645.15 \pm \\
24,400.29\end{array}$ & 0.12 \\
\hline
\end{tabular}

Abbreviations: $S R$, sinus rhythm; $A F$, atrial fibrillation; $n$, number; $B M I$, body mass index; eGFR, estimated glomerular filtration rate; $\mathrm{AH}$, arterial hypertension; ECV, electrical cardioversion; ACEl, angiotensin-converting enzyme inhibitors; ARB, angiotensin II receptor blockers; AAD, antiarrhythmic drugs (Ic/lll class); NTproBNP, N-terminal-pro B-type natriuretic peptide; NT-proANP, N-terminal proatrial natriuretic peptide.

\section{Changes of Echo-Doppler Parameters within I Month After ECV}

LV end-diastolic diameter (LVEDD) decreased from 51.02 \pm 3.11 to $50.40 \pm 3.08 \mathrm{~mm}(P<0.0001)$ and LV EF increased from $54.69 \pm 6.96$ to $56.83 \pm 5.54 \%(P<0.0001)$ after 1 month in SR. The LAVI decreased significantly after 1 month in SR from $51.98 \pm 11.33$ to $45.14 \pm 12.05 \mathrm{~mL} / \mathrm{m}^{2}(P<0.0001)$, which in turn improved significantly LAEF from $26.21 \pm 9.18$ to $38.49 \pm 9.24 \% \quad(P=0.01)$, whereas the antero-posterior LA

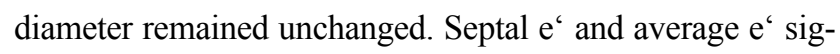
nificantly decreased after 1 month in SR, E wave was not significantly different, and E/e' ratio tended to improve during the follow-up, but the change remained statistically 
Table 2 Baseline Echo-Doppler Parameters of the Study Group

\begin{tabular}{|c|c|c|c|c|}
\hline & Total Cohort n=77 & SR Group n= 39 & AF Group $n=38$ & $\boldsymbol{P}$ \\
\hline LVEDD, mm & $50.52 \pm 3.50$ & $51.02 \pm 3.11$ & $50.00 \pm 3.83$ & 0.23 \\
\hline Left ventricular EF, \% & $54.92 \pm 8.31$ & $54.69 \pm 6.96$ & $55.16 \pm 9.60$ & 0.82 \\
\hline Antero-posterior LA diameter, $\mathrm{mm}$ & $49.03 \pm 3.86$ & $48.93 \pm 4.09$ & $49.14 \pm 3.67$ & 0.82 \\
\hline Maximum LAVI, mL/m² & $52.86 \pm 10.47$ & $51.98 \pm 11.33$ & $53.79 \pm 9.55$ & 0.47 \\
\hline LAEF, \% & $25.07 \pm 8,56$ & $26.21 \pm 9.18$ & $23.86 \pm 7.81$ & 0.25 \\
\hline E wave, $\mathrm{cm} / \mathrm{s}$ & $96.04 \pm 16.74$ & $89.4 I \pm 14.84$ & $103.03 \pm 15.93$ & $<0.0001$ \\
\hline E-deceleration time, $\mathrm{ms}$ & $177.10 \pm 18.73$ & $|8| .01 \pm 18.69$ & $\mid 72.69 \pm 18.05$ & 0.06 \\
\hline Septal e', cm/s & $8.66 \pm 1.21$ & $9.08 \pm 1.15$ & $8.22 \pm 1.12$ & 0.001 \\
\hline Lateral e', cm/s & $12.05 \pm 1.54$ & $12.44 \pm 1.49$ & $11.64 \pm 1.49$ & 0.02 \\
\hline Average $\mathrm{e}^{\prime}, \mathrm{cm} / \mathrm{s}$ & $10.35 \pm 1.28$ & $10.76 \pm 1.24$ & $9.93 \pm 1.19$ & 0.004 \\
\hline E/e' ratio & $9.46 \pm 2.12$ & $8.49 \pm 2.03$ & $10.47 \pm 1.73$ & $<0.0001$ \\
\hline Average $\mathrm{s}^{\prime}, \mathrm{cm} / \mathrm{s}$ & $6.87 \pm 0.77$ & $6.89 \pm 0.86$ & $6.85 \pm 0.67$ & 0.79 \\
\hline$E /\left(e^{\prime} x s^{\prime}\right)$ ratio & $1.4 I \pm 0.35$ & $1.29 \pm 0.37$ & $1.53 \pm 0.29$ & 0.003 \\
\hline
\end{tabular}

Abbreviations: SR, sinus rhythm; AF, atrial fibrillation; LVEDD, left ventricular end diastolic diameter; EF, ejection fraction; LA, left atrial; LAVI, left atrium volume index; LAEF, left atrium emptying fraction; $E$, mitral peak early diastolic filling velocity; e', peak early diastolic mitral annular velocity; s', peak systolic mitral annular velocity; E/(e'x s'), the ratio of mitral peak early diastolic filling velocity (E) to peak early diastolic mitral annular velocity (e') multiplied by peak systolic (s') mitral annular velocity derived by Doppler tissue imaging.

Table 3 Echo-Doppler Parameters After I Month in SR Group

\begin{tabular}{|l|l|l|l|}
\hline & Baseline & Follow-Up & p \\
\hline LVEDD, mm & $51.02 \pm 3.11$ & $50.40 \pm 3.08$ & $<0.000 I$ \\
Left ventricular EF, \% & $54.69 \pm 6.96$ & $56.83 \pm 5.54$ & $<0.000$ I \\
Antero-posterior LA diameter, mm & $48.93 \pm 4.09$ & $45.93 \pm 6.87$ & 0.12 \\
Maximum LAVI, mL/m & $51.98 \pm 11.33$ & $45.14 \pm 12.05$ & $<0.000$ I \\
LAEF, \% & $26.21 \pm 9.18$ & $38.49 \pm 9.24$ & 0.01 \\
E wave, cm/s & $89.41 \pm 14.84$ & $69.96 \pm 19.77$ & 0.57 \\
E-deceleration time, ms & $181.01 \pm 18.69$ & $189.06 \pm 34.87$ & 0.31 \\
Septal e', cm/s & $9.08 \pm 1.15$ & $7.32 \pm 1.25$ & 0.003 \\
Lateral e', cm/s & $12.44 \pm 1.49$ & $10.60 \pm 1.83$ & 0.05 \\
Average e', cm/s & $10.76 \pm 1.24$ & $8.96 \pm 1.47$ & 0.01 \\
E/e' ratio & $8.49 \pm 2.03$ & $7.86 \pm 1.91$ & \\
Average s', cm/s & $6.89 \pm 0.86$ & $7.58 \pm 0.76$ & 0.23 \\
E/(e'xs') ratio & $1.29 \pm 0.37$ & $1.04 \pm 2.78$ & 0.001 \\
\hline
\end{tabular}

Abbreviations: LVEDD, left ventricular end diastolic diameter; EF, ejection fraction; LA, left atrial; LAVI, left atrium volume index; LAEF, left atrium emptying fraction; E, mitral peak early diastolic filling velocity; e', peak early diastolic mitral annular velocity; E/e', the ratio of mitral peak early diastolic filling velocity (E) to peak early diastolic mitral annular velocity ( $\left.e^{\prime}\right)$ by Doppler tissue imaging; s', peak systolic mitral annular velocity; E/(e'xs'), the ratio of mitral peak early diastolic filling velocity (E) to peak early diastolic mitral annular velocity (e') multiplied by peak systolic (s') mitral annular velocity derived by Doppler tissue imaging.

insignificant (from $8.49 \pm 2.03$ to $7.86 \pm 1.91, P=0.23$ ). A decrease of average $e^{6}$ and increase of average $s^{6}$ values led to significant improvement of E/(e'xs') ratio (from 1.29 \pm 0.37 to $1.04 \pm 2,78, P=0.02$ ). All changes of echocardiographic parameters after 1 month in SR group are presented in Table 3.

\section{Predictors of AF Recurrence After ECV}

Binary logistic regression analysis demonstrated that LVEDD $>49.3 \mathrm{~mm}$, NT-proBNP>2000 ng/L, E wave, average $\mathrm{e}^{\prime}, \mathrm{E} / \mathrm{e}^{\prime}$, and $\mathrm{E} /\left(\mathrm{e}^{\prime} \mathrm{xs} \mathrm{s}^{\prime}\right)$ ratios were significant predictors of AF early recurrence after ECV. We developed multivariate models using these potential confounding factors. To minimize the problem of collinearity, E/e' and E/(e'xs') were included in two separate models and $\mathrm{E}$ wave was not included, being already represented within the both derived ratios. Neither continuous nor categorical variables of NT-proANP (expressed as percentiles or cut-off value derived on ROC analysis) were not significantly associated with AF recurrence in the binary logistic regression. Multivariate analysis identified that $E /\left(e^{\prime} x s^{\prime}\right)$ ratio and $\mathrm{E} / \mathrm{e}^{\prime}$ ratio in combination with $\mathrm{LVEDD}>49.3 \mathrm{~mm}$ and 
NT-proBNP $>2000 \mathrm{ng} / \mathrm{L}$ are able to predict early AF recurrence after $\mathrm{ECV}$ in $74 \%$ and $79 \%$ of cases, respectively.

\section{A Comparison Between E/(e' $\left.\times s^{\prime}\right)$ and $E /$ e' Ratios to Predict AF Recurrence After ECV}

$\mathrm{E} /\left(\mathrm{e}^{\prime} \mathrm{xs}\right.$ ') ratio demonstrated a higher contribution in the $\mathrm{AF}$ recurrence prediction model compared to $\mathrm{E} / \mathrm{e}$ ' ratio (for $\mathrm{E} /$ (e'xs') ratio, $\mathrm{HR}=18.94,95 \% \mathrm{CI}=2.39-149.66, P=0.005$; for E/e' ratio, $\mathrm{HR}=1.95, \quad 95 \% \quad \mathrm{CI}=1.31-2.91, \quad P=0.001)$. Univariate and multivariate analyses concerning the variables which predicted the AF recurrence are shown in Table 4. On ROC analysis, E/(e'xs') ratio and E/e' ratio were found to have the largest areas under the curve (AUC) (E/(e'xs') ratio, $\mathrm{AUC}=0.71, P=0.002$; and $\mathrm{E} / \mathrm{e}^{\prime}$ ratio, AUC $=0.75, P<0.0001$ ), with an optimal $\mathrm{E} /\left(\mathrm{e}^{\prime} \mathrm{xs}\right.$ ') ratio cutoff value of 1.15 (sensitivity $97 \%$, specificity $58 \%$ ) and an E/ $\mathrm{e}^{\prime}$ ratio value of 8.79 (sensitivity $89 \%$, specificity $41 \%$ ) for predicting early recurrence of AF after ECV. All ROC curve analysis of independent echocardiography parameters for the prediction of AF early recurrence after ECV are presented in

Table 4 Logistic Regression Analysis, Hazard Ratio for Risk of AF Early Recurrence After ECV

\begin{tabular}{|c|c|c|c|}
\hline \multicolumn{4}{|l|}{ A. Univariate Models } \\
\hline Variables & Hazard Ratio & $95 \% \mathrm{Cl}$ & $\boldsymbol{P}$ \\
\hline$E /\left(e^{\prime} x s^{\prime}\right)$ ratio & 8.17 & $1.57-42.4$ & 0.01 \\
\hline LVEDD $>49.3, \mathrm{~mm}$ & 3.70 & $1.29-10.58$ & 0.02 \\
\hline NT-proBNP>2000, ng/L & 3.28 & $1.01-10.71$ & 0.04 \\
\hline E/e' ratio & 1.74 & $1.27-2.39$ & 0.001 \\
\hline E wave, $\mathrm{cm} / \mathrm{s}$ & 1.06 & I.02-I.II & 0.002 \\
\hline Average $\mathrm{e}^{\prime}, \mathrm{cm} / \mathrm{s}$ & 0.58 & $0.36-0.91$ & 0.02 \\
\hline \multicolumn{4}{|l|}{ B. Multivariate Models } \\
\hline \multicolumn{4}{|c|}{ Model I (Overall Percentage $=74 \%$, Nagelkerke R Square- 0.34 ) } \\
\hline$E /\left(e^{\prime} \times s^{\prime}\right)$ ratio & 18.94 & $2.39-149.66$ & 0.005 \\
\hline LVEDD $>49.3, \mathrm{~mm}$ & 5.54 & $1.58-19.39$ & 0.007 \\
\hline NT-proBNP>2000, ng/L & 4.17 & $1.10-15.72$ & 0.04 \\
\hline \multicolumn{4}{|c|}{ Model 2 (Overall Percentage $=9 \%$, Nagelkerke R Square $=0.43$ ) } \\
\hline LVEDD $>49.3, \mathrm{~mm}$ & 6.22 & $1.69-22.95$ & 0.006 \\
\hline NT-proBNP>2000, ng/L & 4.08 & $1.06-15.73$ & 0.04 \\
\hline $\mathrm{E} / \mathrm{e}^{\prime}$ ratio & 1.95 & $|.31-2.9|$ & 0.001 \\
\hline
\end{tabular}

Abbreviations: $\mathrm{Cl}$, confidence interval; $\mathrm{E} /\left(\mathrm{e}^{\prime} \mathrm{xs}\right)$, the ratio of mitral peak early diastolic filling velocity $(\mathrm{E})$ to peak early diastolic mitral annular velocity (e') multiplied by peak systolic (s') mitral annular velocity derived by Doppler tissue imaging; LVEDD, left ventricular end diastolic diameter; NT-proBNP, N-terminal-pro B-type natriuretic peptide; $E / e^{\prime}$, the ratio of mitral peak early diastolic filling velocity $(E)$ to peak early diastolic mitral annular velocity (e') by Doppler tissue imaging; E, mitral peak early diastolic filling velocity; e', peak early diastolic mitral annular velocity.
Table 5. Figure 2 shows the ROC curves for the prediction of early (at 1 month) recurrence of AF.

\section{Discussion}

The key findings of this report are three-fold:

1. $\mathrm{E} /\left(\mathrm{e}^{\prime} \mathrm{xs} \mathrm{s}^{\prime}\right)$ and $\mathrm{E} / \mathrm{e}^{\prime}$ ratios are possible predictive factors of early AF recurrence after ECV.

2. Complex assessment of NT-proBNP level and LVEDD increases prediction ability of early AF recurrence of $\mathrm{E} /\left(\mathrm{e}^{\prime} \mathrm{xs}\right.$ ') ratio almost 3-fold, whereas $\mathrm{E} / \mathrm{e}^{\prime}$ power remains unchanged.

3. Value of $e^{6}$ and derived ratios to identify diastolic dysfunction in patients with $\mathrm{AF}$ is questionable.

The LA size is considered as one of the key parameters to determine the risk of AF recurrence after SR restoration procedures. ${ }^{7,18,19}$ Recent studies revealed that impaired LA function can be a more valuable predictor of possible AF recurrence compared to structural LA enlargement alone. ${ }^{19,20}$ Nevertheless, in our study population, we did not notice any significant differences of LAVI and LAEF between SR and AF groups. This could be explained by the fact that LAVI values in our study tended to be higher compared to studies which confirmed LAVI prognostic value for AF recurrence (LAVI of total cohort 52.86 $\left.\pm 10.47 \mathrm{~mL} / \mathrm{m}^{2}\right){ }^{7,18,19}$ Fornengo et $\mathrm{al}^{5}$ also demonstrated that LAVI and total LAEF failed in predicting the risk of AF recurrence in patients with moderate-to-severe LA enlargement (LAVI $\geq 34 \mathrm{~mL} / \mathrm{m}^{2}$ ). Our results illustrate that the LAVI and LAEF also did not provide supplementary information to indices of diastolic function parameters.

The LA emptying pattern is strongly influenced by the LV filling pressures. Diastolic LV dysfunction over time can lead to progressive LA enlargement, gradually decreased LA function, and electrical instability. ${ }^{21}$ According to this assumption, LV diastolic dysfunction may play a pivotal role in the development and maintenance of AF. In the present study, high $\mathrm{E} / \mathrm{e}^{\text {c }}$ and low $\mathrm{e}^{\text {c }}$ values were significantly associated with $\mathrm{AF}$ recurrence, which is in line with the previous reports. ${ }^{5-7}$

Relaxation and contraction are equivalent parts of a continuous cardiac cycle, where systolic and diastolic abnormalities have a variable contribution to the LV impairment. ${ }^{22} \mathrm{The} \mathrm{E} /\left(\mathrm{e}^{\prime} \mathrm{xs}\right.$ ') ratio combines an index of diastolic function (E/e') and a marker of LV systolic function (s'). It revealed a good correlation with invasively measured LV end-diastolic pressure regardless of LV EF and present 
Table 5 ROC Curve Analysis of Echocardiography Parameters for the Prediction of AF Early Recurrence After ECV

\begin{tabular}{|l|l|l|l|l|l|}
\hline Parameters & Cutoff Value & Sensitivity (\%) & Specificity (\%) & AUC & $P$ \\
\hline E wave, cm/s & 96.00 & 70 & 30 & 0.71 & 0.001 \\
Average e', cm/s & 10.13 & 65 & 33 & 0.67 & 0.01 \\
E/e' ratio & 8.79 & 89 & 41 & 0.75 & $<0.0001$ \\
E/(e'Xs') ratio & 1.15 & 97 & 58 & 0.72 & 0.002 \\
\hline
\end{tabular}

Abbreviations: ROC, receiver operating characteristic; AUC, area under the curve; E, mitral peak early diastolic filling velocity; e', peak early diastolic mitral annular velocity; E/e', the ratio of mitral peak early diastolic filling velocity $(E)$ to peak early diastolic mitral annular velocity (e') by Doppler tissue imaging; E/(e'xs'), the ratio of mitral peak early diastolic filling velocity (E) to peak early diastolic mitral annular velocity (e') multiplied by peak systolic (s') mitral annular velocity derived by Doppler tissue imaging.

regional dysfunction. ${ }^{23}$ Previous studies demonstrated the usefulness of the E/(e'xs') ratio to predict new-onset $A F$ and $\mathrm{AF}$ recurrence after $\mathrm{ECV}{ }^{8,10}$ Univariate and multivariate analysis of our data showed that the E/(e'xs') ratio was a significant predictor of early AF recurrence after ECV and demonstrated higher hazard ratios than $\mathrm{E} / \mathrm{e}$ ' ratio. ROC analysis revealed that AUCs of E/(e'xs') and E/e' ratios were similar: $\mathrm{AUC}=0.71$ and $\mathrm{AUC}=0.75$, respectively. he TAUC of the E/(e'xs') ratio showed slightly better sensitivity and specificity than the AUC of the E/e' ratio (sensitivity $97 \%$ for $\mathrm{E} /\left(\mathrm{e}^{\prime} \mathrm{xs}\right.$ ') compared to $89 \%$ for E/e' and specificity $58 \%$ for E/(e'xs') compared to $41 \%$ for E/e'). Regarding out study the prediction accuracy of the $\mathrm{E} /\left(\mathrm{e}^{\prime} \mathrm{xs}\right.$ ') ratio for $\mathrm{AF}$ recurrence was comparable to the E/e' ratio, whereas Ari et al ${ }^{10}$ demonstrated an obvious advantage of E/(e'xs') to predict shortterm recurrence of AF after ECV comparing to the E/e' ratio. Larger studies are required to be performed to verify the superiority of E/(e'xs') ratio against conventional echocardiographic variables for diagnosing a diastolic dysfunction in AF patients.

Many clinical factors, such as preprocedural natriuretic peptides levels, inflammatory factors, various echocardiographic measures of LA morphology and function were investigated as possible predictors of AF recurrence. Regarding controversial results and the heterogenecity of the patient population that suffer from AF there is no single predictor of AF recurrence to optimize patients' selection for SR restoration by ECV. In our study baseline preprocedural NT-proBNP level alone had no prognostic value, differently from what has been recently confirmed by the two meta-analyses. ${ }^{24,25}$ AF by itself is related to increased levels of plasma natriuretic peptides and significant reduction or normalization is observed after the reversion to SR. ${ }^{26,27}$ Latest studies revealed that BNP levels

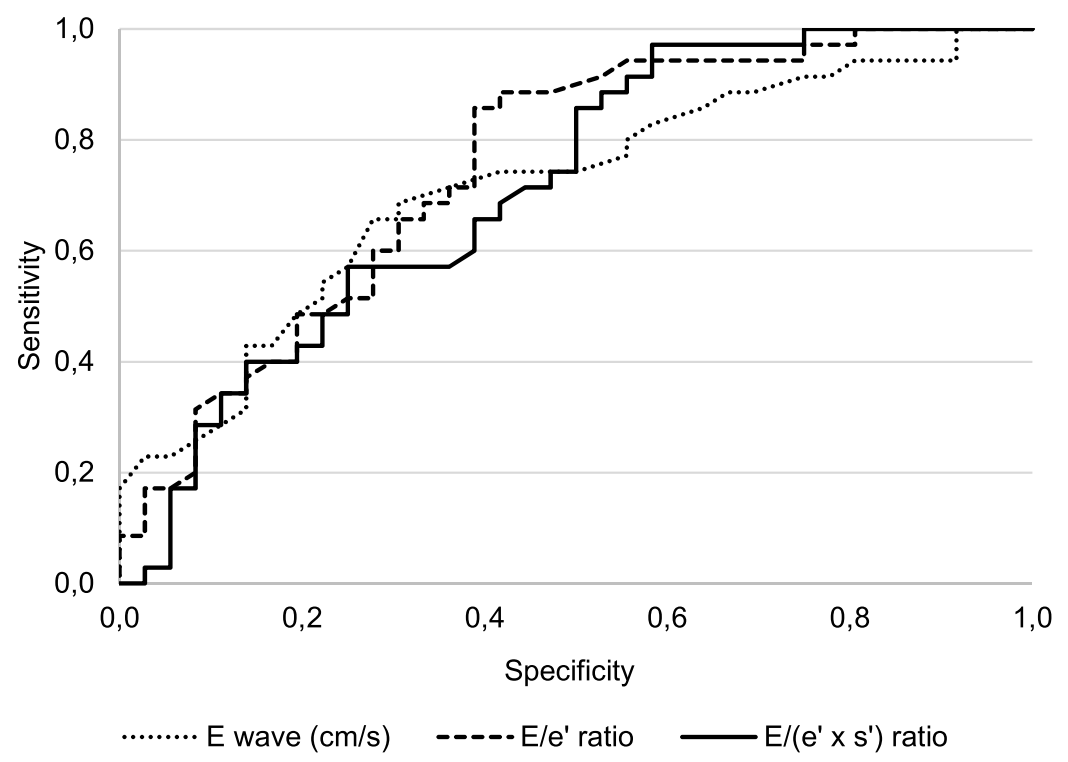

Figure 2 ROC curves for the prediction of early (within I month) recurrence of AF.

Abbreviations: ROC, receiver operating characteristic; AF, atrial fibrillation; E, mitral peak early diastolic filling velocity; E/e', the ratio of mitral peak early diastolic filling velocity (E) to peak early diastolic mitral annular velocity (e') by Doppler tissue imaging; $E /\left(e^{\prime} x s^{\prime}\right)$, the ratio of mitral peak early diastolic filling velocity (E) to peak early diastolic mitral annular velocity (e') multiplied by peak systolic (s') mitral annular velocity derived by Doppler tissue imaging. 
were significantly positively related to E/e' in heart failure with preserved EF patients. ${ }^{28}$ Analysis of our data demonstrates that complex evaluation of diastolic function combining NT-proBNP levels and echocardiographic variables have better prognostic value compared to each component considered separately. Complex evaluation increased the contribution of $\mathrm{E} /\left(\mathrm{e}^{\prime} \mathrm{xs}\right.$ ') ratio to predict $\mathrm{AF}$ recurrence from $\mathrm{HR}=8.17$ in univariate analysis to $\mathrm{HR}=18.94$ in the complex model, whereas the $\mathrm{E} / \mathrm{e}$ ' ratio contribution remained similar: $H R=1.74$ in univariate analysis and $\mathrm{HR}=1.95$ in the model.

In patients with AF, the cut-off values of Doppler assessment of LV diastolic function is almost the same as in patients with SR. ${ }^{3}$ However, previous studies stated inconsistent results show tissue Doppler velocities changes after ECV. Melek et $\mathrm{al}^{29}$, found no significant changes in e' velocities 1 month after ECV, in comparison, Fung et $\mathrm{al}^{30}$ demonstrated the increasement of $\mathrm{e}^{\prime}$ in 1 month following ECV. In our study, septal, lateral, and average e' decreased, whereas E/e' increased, but the change remained statistically insignificant. This result is similar to the findings of Park et al, ${ }^{12}$ where e' was significantly higher in AF compared with during SR in the same patients. Restoration of atrial mechanical contraction following ECV improved LV systolic function, assessed by s', and together had a larger impact on LV function assessed by E/(e'xs') ratio that statistically significantly improved. These findings suggest that the e' and derived ratios that include spectral tissue Doppler variables might be inaccurate and should be used with caution in order to identify diastolic dysfunction in patients with AF. The latest systematic review by Nauta et $\mathrm{al}^{31}$ also revealed that E/e' showed the best, albeit modest correlation with invasive measurements of elevated LV filling pressure.

\section{Limitations}

The present study has several limitations. First, it was a single-center study with a relatively small number of patients. Second, we chose to perform echocardiography within 24 hours after ECV in regular R-R intervals of SR, thus obviously limiting the clinical implications of our findings on the everyday clinical practice, when routine echocardiography is not performed to patients with AF. Third, our results can be limited by a relatively short follow-up period of 1 month, which can result in less significant results. And, finally, patients with reduced left ventricular ejection fraction were not included in our cohort and we did not compare the results between certain groups of patients according to their left ventricular ejection fraction. Larger studies with a longer follow-up period are needed to be performed to establish our initial results for certain groups of patients.

\section{Conclusion}

E/(e'xs') and E/e' ratios are comparable to predict early AF recurrence after ECV in patients with persistent AF. In the AF recurrence prediction model, which included a higher NT-proBNP level, larger LVEDD, and spectral tissue Doppler-derived ratios, E/(e'xs') ratio demonstrated a higher contribution compared to the E/e' ratio. The e' decreased significantly within 1-month after ECV for persistent AF patients with normal LV EF. Thus, the value of $\mathrm{e}^{6}$ and derived ratios to detect diastolic dysfunction in patients with AF are questionable.

\section{Acknowledgments}

Rasa Karaliute, Justina Jureviciute, Vaida Mizariene, Giedre Baksyte, Tomas Kazakevicius, and Ausra Kavoliuniene are members of the Kaunas Region Society of Cardiology. The Kaunas Region Society of Cardiology funded the article publishing charge.

\section{Disclosure}

Ausra Kavoliuniene reports personal fees from Bayer and Novartis Pharma, outside the submitted work. The authors report no other conflicts of interest in this work.

\section{References}

1. Sharma PS, Callans DJ. Treatment considerations for a dual epidemic of atrial fibrillation and heart failure. J Atr Fibrillation. 2013;6(2):740.

2. Pieske B, Tschöpe C, de Boer RA, et al. How to diagnose heart failure with preserved ejection fraction: the HFA-PEFF diagnostic algorithm: a consensus recommendation from the Heart Failure Association (HFA) of the European Society of Cardiology (ESC). Eur Heart $J$. 2019;40(40):3297-3317.

3. Nagueh SF, Smiseth OA, Appleton CP, et al. Recommendations for the evaluation of left ventricular diastolic function by echocardiography: an update from the American Society of Echocardiography and the European Association of Cardiovascular Imaging. Eur Heart $J$ Cardiovasc Imaging. 2016;17(12):1321-1360.

4. Arques S. Why not integrate the spectral tissue Doppler E/(e'xs') in the multiparametric assessment of cardiovascular diseases by transthoracic Doppler echocardiography? Ann Cardiol Angeiol (Paris). 2019;68(5):358-362. doi:10.1016/j.ancard.2019.07.012

5. Fornengo C, Antolini M, Frea S, et al. Prediction of atrial fibrillation recurrence after cardioversion in patients with left-atrial dilation. Eur Heart J Cardiovasc Imaging. 2015;16(3):335-341. doi:10.1093/ehjci/ jeu193

6. Okura H, Takada Y, Kubo T, et al. Tissue Doppler-derived index of left ventricular filling pressure, E/E', predicts survival of patients with non-valvular atrial fibrillation. Heart. 2006;92(9):1248-1252. doi:10.1136/hrt.2005.082594 
7. Wałek P, Sielski J, Starzyk K, et al. Echocardiographic assessment of left atrial morphology and function to predict maintenance of sinus rhythm after electrical cardioversion in patients with non-valvular persistent atrial fibrillation and normal function or mild dysfunction of left ventricle. Cardiol J. 2020;27(3):246-253. doi:10.5603/CJ. a2019.0068

8. Mornos C, Petrescu L, Cozma D, et al. A new tissue doppler index in predicting future atrial fibrillation in patients with heart failure. Arq Bras Cardiol. 2011;97(6):468-477.

9. Mornos C, Petrescu L, Cozma D, et al. A new tissue Doppler index to predict cardiac death in patients with heart failure. Arq Bras Cardiol. 2014;102(1):19-29.

10. Ari H, Ari S, Sarigül OY, et al. A novel index combining diastolic and systolic tissue doppler parameters for prediction of atrial fibrillation recurrence. Echocardiography. 2016;33(7):1009-1015. doi:10.1111/echo.13212

11. Kotecha D, Mohamed M, Shantsila E, et al. Is echocardiography valid and reproducible in patients with atrial fibrillation? A systematic review. Europace. 2017;19(9):1427-1438. doi:10.1093/europace/eux027

12. Park K, Park TH, Kim SJ, et al. Changes in mitral annular velocities after cardioversion of atrial fibrillation. Echocardiography. 2018;35 (11):1782-1787. doi:10.1111/echo.14139

13. Fraser CG. Quality specifications for imprecision of B-type natriuretic peptide assays. Clin Chem. 2005;51(7):1307-1309. doi:10.1373/ clinchem.2005.050393

14. Levey AS, Stevens LA, Schmid $\mathrm{CH}$, et al. A new equation to estimate glomerular filtration rate. Ann Intern Med. 2009;150 (9):604-612. doi:10.7326/0003-4819-150-9-200905050-00006

15. Kirchhof P, Benussi S, Kotecha D, et al. 2016 ESC Guidelines for the management of atrial fibrillation developed in collaboration with EACTS. Europace. 2016;18(11):1609-1678.

16. Lang RM, Badano LP, Mor-Avi V, et al. Recommendations for cardiac chamber quantification by echocardiography in adults: an update from the American Society of Echocardiography and the European Association of Cardiovascular Imaging. Eur Heart J Cardiovasc Imaging. 2015;16(3):233-270.

17. Blume GG, Mcleod CJ, Barnes ME, et al. Left atrial function: physiology, assessment, and clinical implications. Eur $J$ Echocardiogr. 2011;12(6):421-430. doi:10.1093/ejechocard/jeq175

18. Toufan M, Kazemi B, Molazadeh N. The significance of the left atrial volume index in prediction of atrial fibrillation recurrence after electrical cardioversion. J Cardiovasc Thorac Res. 2017;9(1):54-59. doi:10.15171/jcvtr.2017.08

19. Luong CL, Thompson DJ, Gin KG, et al. Usefulness of the atrial emptying fraction to predict maintenance of sinus rhythm after direct current cardioversion for atrial fibrillation. Am J Cardiol. 2016;118 (9):1345-1349.
20. Chou CC, Lee HL, Chang PC, et al. Left atrial emptying fraction predicts recurrence of atrial fibrillation after radiofrequency catheter ablation. PLoS One. 2018;13(1):e0191196. doi:10.1371/journal. pone.0191196

21. Mehrzad R, Rajab M, Spodick DH. The three integrated phases of left atrial macrophysiology and their interactions. Int $J \mathrm{Mol} \mathrm{Sci}$. 2014;15(9):15146-15160.

22. Yip G, Wang M, Zhang Y, et al. Left ventricular long axis function in diastolic heart failure is reduced in both diastole and systole: time for a redefinition? Heart. 2002;87(2):121-125. doi:10.1136/heart.87.2.121

23. Mornos C, Cozma D, Rusinaru D, et al. A novel index combining diastolic and systolic Tissue Doppler parameters for the non-invasive assessment of left ventricular end-diastolic pressure. Int J Cardiol. 2009;136(2):120-129. doi:10.1016/j.ijcard.2008.04.029

24. Zografos T, Maniotis C, Katsivas A, et al. Relationship between brain natriuretic peptides and recurrence of atrial fibrillation after successful direct current cardioversion: a meta-analysis. Pacing Clin Electrophysiol. 2014;37(11):1530-1537.

25. Xu X, Tang Y. Relationship between brain natriuretic peptide and recurrence of atrial fibrillation after successful electrical cardioversion: an updated meta-analysis. Braz J Cardiovasc Surg. 2017;32 (6):530-535.

26. Wozakowska-Kaplon B. Changes in plasma natriuretic peptide levels in patients with atrial fibrillation after cardioversion. Int $J$ Cardiol. 2010;144(3):436-437. doi:10.1016/j.ijcard.2009.03.085

27. Higa C, Ciambrone G, Donato MS, et al. NT-Pro-BNP levels performance before and after reversion to sinus rhythm in patients with preserved ventricular function. Medicina (B Aires). 2011;71(2):146-150.

28. Islam MN, Chowdhury MS, Paul GK, et al. Association of diastolic dysfunction with N-terminal Pro-B-type natriuretic peptide level in heart failure patients with preserved ejection fraction. Mymensingh Med J. 2019;28(2):333-346.

29. Melek M, Birdane A, Goktekin O, et al. The effect of successful electrical cardioversion on left ventricular diastolic function in patients with persistent atrial fibrillation: a tissue Doppler study. Echocardiography. 2007;24(1):34-39. doi:10.1111/j.15408175.2007.00347.x

30. Fung KC, Tan HC, Kritharides L. Acute reductions in ventricular myocardial tissue velocities after direct current cardioversion of atrial fibrillation. $J$ Am Soc Echocardiogr. 2003;16(6):656-663. doi:10.1016/S0894-7317(03)00178-0

31. Nauta JF, Hummel YM, van der Meer P, et al. Correlation with invasive left ventricular filling pressures and prognostic relevance of the echocardiographic diastolic parameters used in the 2016 ESC heart failure guidelines and in the 2016 ASE/EACVI recommendations: a systematic review in patients with heart failure with preserved ejection fraction. Eur J Heart Fail. 2018;20(9):1303-1311.
Clinical Interventions in Aging

\section{Publish your work in this journal}

Clinical Interventions in Aging is an international, peer-reviewed journal focusing on evidence-based reports on the value or lack thereof of treatments intended to prevent or delay the onset of maladaptive correlates of aging in human beings. This journal is indexed on PubMed Central, MedLine, CAS, Scopus and the Elsevier
Bibliographic databases. The manuscript management system is completely online and includes a very quick and fair peer-review system, which is all easy to use. Visit http://www.dovepress.com/ testimonials.php to read real quotes from published authors. 